\title{
Ovarian conservation versus removal at the time of hysterectomy for benign gynecological diseases
}

\author{
Tarafdar Runa Laila ${ }^{1}$, Sheikh Salahuddin Ahmed ${ }^{2}$, Khairun Nahar ${ }^{1}$, \\ Parveen Akhter Shamsunnahar ${ }^{1}$, Sharmeen Mahmood ${ }^{1}$, Saleha Begum Chowdhury ${ }^{1}$ \\ ${ }^{1}$ Bangabandhu Sheikh Mujib Medical University, Dhaka, Bangladesh \\ ${ }^{2}$ Bangladesh Institute of Health Sciences (BIHS), Dhaka, Bangladesh \\ Email address: \\ trlaila@yahoo.com (T. R. Laila)
}

To cite this article:

Tarafdar Runa Laila, Sheikh Salahuddin Ahmed, Khairun Nahar, Parveen Akhter Shamsunnahar, Sharmeen Mahmood, Saleha Begum Chowdhury. Ovarian Conservation Versus Removal at the Time of Hysterectomy for Benign Gynecological Diseases. American Journal of Clinical and Experimental Medicine. Vol. 2, No. 2, 2014, pp. 36-41. doi: 10.11648/j.ajcem.20140202.15

\begin{abstract}
Hysterectomy for benign gynecological diseases is a common surgical procedure. Prophylactic bilateral oophorectomy is often recommended concurrent with hysterectomy to decrease the risk of ovarian cancer. Oophorectomy before menopause leads to an abrupt decrease in endogenous estrogen and androgen production leading to different health problems. So women undergoing hysterectomy for benign gynecological diseases are presented with the choice of ovarian conservation or removal. The purpose of this review article is to summarize and critically evaluate the existing evidences regarding the impact of ovarian conservation versus removal on specific health issues of the individual. Information was collected by searching pub med for related studies, abstracts and articles. Studies have shown that benefits of elective oophorectomy also include reduction of breast cancer and development of residual ovary syndrome. On the other hand bilateral oophorectomy is associated with increased risk of cardiovascular disease, osteoporosis, cognitive impairment, dementia, depression, anxiety and decreased sexual function. Estrogen was commonly prescribed after bilateral oophorectomy to treat menopausal symptoms. But recent studies have shown more harm than benefit in postmenopausal hormone therapy. So the decision making regarding elective oophorectomy at the time of hysterectomy for benign indications has become complex. The approach to such decision will include counseling regarding risks and benefits of the procedure and shared decision making between the clinician and the patient. The beneficial effect on ovarian cancer must be weighed against the risks of ovarian hormone withdrawal.
\end{abstract}

Keywords: Bilateral Oophorectomy, Hysterectomy, Ovarian Cancer

\section{Introduction}

Inspite of newer techniques in the treatment of menstrual disorders, hysterectomy for benign gynecological diseases is a common surgical procedure. Hysterectomy rate vary greatly internationally from 55 per 10,000 in North America, 28 per 10,000 in Britain to 10 per 10,000 in Denmark [1]. Hysterectomy rates vary within countries according to both patient related factors such as race, socioeconomic condition, education status, attitude towards surgery as well as training and practice of the surgeon [2]. Now-a-days the hysterectomy rate to treat menstrual disorder has fallen worldwide due to increased availability of new modalities of treatment like endometrial ablation and levonorgestrel releasing intrauterine devices [3].

During hysterectomy the ovaries can either be removed or retained. Oophorectomy does not add significantly to the duration or immediate complications of the procedure but may have significant effect on both short and long term health. Elective bilateral salpingo-oophorectomy (BSO) is offered to women 45 years or older at the time of hysterectomy to prevent the development of ovarian cancer, a rare but highly morbid disease with a $1.4 \%$ life time risk [4]. Bilateral salpingo-oophorectomy is performed in $40 \%$ benign hysterectomies among women aged 40 to 44 years; $60 \%$ among women aged 45 to 50 years and $78 \%$ among women aged 50 to 55 years $[5,6]$. Previously estrogen was prescribed to treat the menopausal symptoms. However since the Women's Health Initiative randomized trials have shown more harm than benefit associated with 
postmenopausal hormone therapy (HT) there is a dramatic decline in the use of HT [7]. As a result the practice of performing elective BSO with hysterectomy has become more controversial.

The objective of this review article is to evaluate the existing evidences regarding the impact of ovarian conservation versus removal on specific health issues of an individual like ovarian cancer, coronary heart disease (CHD), sexual and cognitive function, and osteoporosis. It also critically reviews the current recommendations regarding the conservation or removal of ovaries during hysterectomy.

\section{Methodology}

Pub Med was searched in 2013 for all English language publications including the search terms, "Ovarian conservation versus removal at the time of hysterectomy for benign gynecological diseases" from 1990 onwards. The full articles and the abstracts that were thought to be potentially relevant were studied. The references in those articles were also checked for additional information.

\section{Surgical Effect on Ovarian Function}

The ovary is an endocrine organ having physiological effects during the postmenopausal as well as the reproductive years. Surgical menopause results in an abrupt drop in estrogen level, an abrupt rise in follicle stimulating hormone (FSH) level and the complete cessation of ovarian hormone production including androgen, estradiol and progesterone $[8,9]$.

Postmenopausal ovaries continue to be hormonally active for many years beyond menopause producing androgen including testosterone, androstenedion and dehydroepiandrosterone (DHEA) as well as small amount of estradiol and estrone [10]. Although estrogen secretion by postmenopausal ovary eventually ceases, ovarian secretion of small amount of testosterone and the androgen precursor DHEA may continue into the eighth decade of life $[8,10]$. Extraovarian sources of sex steroids throughout life include the adrenal glands and the widespread target tissues including fat, where the aromatization of androgen to estrogen occurs. The extent to which extra ovarian sex steroid hormone synthesis differs between women who have experienced natural versus surgical menopause has been debated but it appears that total androgen levels remain lower after surgical menopause than after natural one.

It is found that $35 \%$ of patients undergoing unilateral oophorectomy had impairment of ovarian function as measured by FSH, LH, and 17- $\beta$ estradiol levels upto six months following hysterectomy; while no patients six months following hysterectomy with bilateral ovarian conservation showed such failure [11]. This suggests that conservation of both ovaries has better outcome.

\section{Benefits of Elective Oophorectomy}

\subsection{Reduction of Ovarian Cancer}

Reduction in the future risk of ovarian cancer is the single most common reason for normal ovaries to be removed at the time of hysterectomy. A combination of factors including the lack of a proven efficient early screening test and non specific presenting symptoms mean that ovarian cancer tends to be diagnosed at a more advanced stage than other gynecological cancer. Most patients present with advanced disease for which 5- year survival is $28 \%$ [12]. Sometimes the decision is left until the time of surgery with the plan to retain the ovaries if they appear normal. It is not known whether a surgeon's impression of normality equates reliably to histologically confirmed ovarian pathology. Clinical experience suggests that there is likely to be a high chance of false positive. Since pelvic ultrasound is very commonly performed prior to hysterectomy and has a high sensitivity in detecting ovarian pathology, it seems relatively unlikely in modern surgical practice that significant ovarian pathology will be clinically apparent for the first time at the time of hysterectomy.

$\mathrm{BSO}$ is an important risk reduction strategy for women who are at high risk of ovarian cancer. The life time risk of ovarian cancer in the general population is 1.4 percent compared with 13 to 46 percent in women with BRCA 1 or BRCA 2 gene mutations and 3 to 14 percent in those with Lynch syndrome (hereditary non-polyposis colorectal cancer syndrome) [13]. Salpingo-oophorectomy in these women is risk reducing and not elective.

Surgical procedures other than BSO may reduce the risk of ovarian cancer without resulting in surgical menopause. Procedures that interrupt or remove the fallopian tubes appear to reduce the risk of ovarian cancer but not to the same extent as BSO. Potential mechanism for the protective effect of these procedures include: (1) eliminating lesions that may have originated in the fallopian tubes (2) limiting the potential for upward migration of carcinogens through the vagina, cervix and fallopian tubes into the peritoneal cavity and (3) providing a cancer screening effect [14].

\subsection{Reduction of Breast Cancer}

Oophorectomy appears to reduce the risk of breast cancer in women who are 45 years old or younger at the time of surgery. The reduced risk of breast cancer is likely due to reduced exposure to estrogen from the premenopausal ovary. The Nurses' Health Study was a large prospective study that reported a reduction in breast cancer risk in young women who underwent oophorectomy. A significant reduction in breast cancer incidence was found only in women who underwent oophorectomy at less than 45 years old (222 versus 315 cases per 100,000 person- years; hazard ratio (HR) of $0.6,95 \%$ CI 0.5-0.7) [15]. In contrast the Women's Health Initiative Observational Study found no significant reduction in breast cancer risk in women who underwent oophorectomy compared with those who did not undergo 
oophorectomy; these findings can be explained by significant limitations in the study design [12].

\subsection{Prevention of Residual Ovary Syndrome}

Reoperation done for ovarian pathology termed residual ovary syndrome occurred in 3 to 4 percent of women who retained one or both ovaries after hysterectomy [16]. Preoperative pelvic pain and adhesions are risk factors for this syndrome. In a study including 1265 women, it is found that the frequency of residual ovary syndrome was twice as high in women who had one ovary preserved rather than both ( 7.6 versus 3.6 percent) [17]. In addition residual ovary syndrome was more common in women who underwent hysterectomy at a younger age possibly because of the longer post procedure ovarian function with a greater opportunity for functional ovarian pathology. In women who require oophorectomy subsequent to hysterectomy, laparoscopic techniques have reduced the surgical morbidity of such procedures. One small study reported that 32 of 35 women with a prior hysterectomy had successful laparoscopic oophorectomy [18]. Thus minimally invasive surgery will provide a solution for most women who develop ovarian pathology following hysterectomy. So the possible need for subsequent surgery does not appear to justify the deleterious long term consequences of BSO for most women undergoing hysterectomy.

\section{Risks of Elective Oophorectomy}

\subsection{Increased Cardiovascular Morbidity and Mortality}

Cardiovascular disease (CVD) especially coronary heart disease (CHD) is a leading cause of death in older women and the rate increases with menopause [19]. The reasons for this are not fully known but may relate to an accelerated rise in cholesterol level, blood pressure and insulin which primarily relate to increased body weight following the menopause transition [20]. It remains unclear whether hormone therapy (HR) modifies this process or the clinical CVD risk. A large randomized controlled trial failed to show any impact of unopposed conjugated estrogen on CVD in older post menopausal women [7]. So retention of ovarian function with endogenous estrogen may be the best way to reduce risk of CHD. In the Women's Health Initiative study, it was shown that oophorectomy and hysterectomy were associated with 2 fold increased risk of coronary artery calcification compared with those whose ovaries were retained [21]. A recent meta analysis demonstrated that natural menopause did not increase the risk of CVD [relative risk (RR):1.14, 95\% confidence interval (CI): 0.86-1.5] but oophorectomy even at a mean age of 50 years appeared to increase the risk (RR: 2.62, 95\% CI 2.05-3.35) and oophorectomy at younger than 50 years had a substantial negative impact on CVD ((RR: $4.45,95 \%$ CI 2.56- 8.10) [22]. A Danish cohort study also shows that the rates of ischemic heart disease were 7 fold higher in women with a history of oophorectomy younger than 45 years [23].

\subsection{Decreased Cognitive Function}

Oophorectomy prior to menopause appears to be associated with an increased risk of cognitive impairment or dementia as well as parkinsonism. The Mayo Clinic Cohort Study of oophorectomy and aging shows that women who underwent bilateral oophorectomy before menopause compared with those who did not undergo oophorectomy, a significant increase in the risk of cognitive impairment or dementia occurred in those who were younger than 48 years at the time of surgery and who did not take estrogen therapy from time of surgery through age 50 years (HR $1.89,95 \% \mathrm{C}$ I 1.27-2.83) [24]. Neurocognitive performance was worse when oophorectomy occurred at younger ages and worse with a greater decline in estradiol level but it was better when hormone therapy was initiated after oophorectomy. In the Mayo Clinic Cohort Study of oophorectomy and aging, women who underwent bilateral oophorectomy before the age of menopause had an increased risk of parkinsonism compared with no oophorectomy (2.6 versus 1.2 percent) and the risk increases with younger age at oophorectomy [24, 25]. Most current international guidelines advise taking HT until the average age of natural menopause (around 51 years) [26].

\subsection{Increased risk of Depression and Anxiety}

Some data suggest that early bilateral oophorectomy at the time of hysterectomy is associated with an increased risk of depression and anxiety [27, 28]. The Mayo Clinic Cohort Study of oophorectomy and aging reported that women, who had bilateral oophorectomy compared with those who had retained their ovaries, had a significant increase in the onset of symptoms of depression or anxiety [27]. The median time from oophorectomy to the onset of symptom of depression and anxiety was approximately 14 years but differences in the rates of occurrence of anxiety and depression were observed within three years following hysterectomy and persisted throughout more than 30 years of follow-up [29].

\subsection{Impaired Sexual Function}

The evaluation of quality of life, wellbeing and sexual function following surgical menopause is complex and will partly depend on preoperative characteristics, the indications for hysterectomy and oophorectomy and the specific procedure performed. Removal of the ovaries generally results in around $50 \%$ reduction in circulating testosterone levels [30]. Circulating testosterone level appears to contribute to sexual desire in women. Prospective studies suggest that retaining the ovaries at the time of hysterectomy is associated with improved libido and superior sexual function compared with those who undergo oophorectomy [31]. Adverse effect of bilateral oophorectomy can involve several different domains of sexual function including libido, arousal and orgasm [30]. In one retrospective study it is found that women, who had undergone bilateral oophorectomy during hysterectomy, reported significantly decreased sexual satisfaction postoperatively despite 
estrogen treatment compared to those who have their ovaries preserved [32]. A recent systematic review concluded that adding testosterone to estrogen therapy may provide additional improvements in wellbeing in some women, but only at supra physiological levels of total testosterone and physiological levels of free testosterone [33]. However the safety of testosterone alone or in combination with estrogen is not established.

\subsection{Increased risk of Osteoporosis}

Oophorectomy before age 45 is a well-established risk-factor for osteoporosis. In addition, even in women who undergo bilateral oophorectomy after natural menopause, the risk of osteoporotic fracture may be increased compared with women with intact ovaries. The increase in fracture risk among women who underwent bilateral oophorectomy after natural menopause is consistent with the hypothesis that androgens produced by the postmenopausal ovary are important for endogenous estrogen production that protects against fractures.

\section{Estrogen Therapy after Oophorectomy}

Estrogen was commonly prescribed after BSO to treat the menopausal symptoms and substitute for the loss of endogenous ovarian hormones. However the Women's Health Initiative randomized trials demonstrated that conjugated equine estrogen (CEE) does not appear to affect the risk of heart disease but increases the risk of stroke. The small but persistent increase in systolic blood pressure in women taking CEE is one possible contributor to this effect because relatively small difference in systolic blood pressure have been positively associated with differences in stroke [34-35]. However it reduces the risk of hip and other fractures. In a study it is found that there is a modest increase in breast cancer risk with estrogen alone while others have found little or no effect of estrogen alone on breast cancer risk [36]. It may be because the effect of CEE alone on breast density is minimal [37]. So these data demonstrate no overall benefit of CEE for chronic disease prevention after bilateral oophorectomy. The current US Food and Drug Administration recommendation for post menopausal women is to use CEE only for menopausal symptoms at the smallest effective dose for the shortest possible time [38].

\section{Discussion}

All women planning hysterectomy should be assessed for risk factors for ovarian and breast cancer. In addition, an appropriate evaluation for extrauterine pelvic pathology should be performed.

The first step is to determine whether the choice regarding oophorectomy is truly elective. Bilateral oophorectomy is associated with a small absolute cancer risk reduction for women who are at an average risk of ovarian or breast cancer.
Ovarian cancer is not a common disease and hysterectomy itself provides a small preventive effect. For women who have already experienced natural menopause, elective bilateral oophorectomy may be health neutral. A decision analysis based upon a comprehensive review of observational data concluded that ovarian conservation at time of benign hysterectomy until age 65 years benefits long term survival and that the risks and benefits of elective oophorectomy approximate each other after age 65 years [39].

The American college of Obstetrics \& Gynecology (ACOG) advises strong consideration of ovarian conservation at time of hysterectomy in premenopausal women who are not at an increased genetic risk of ovarian cancer [40]. They advise consideration of oophorectomy at time of hysterectomy in women who are post menopausal or who have a condition that may benefit from oophorectomy (endometriosis, pelvic inflammatory disease, chronic pelvic pain). Oophorectomy is reasonable for women who place a high priority on cancer prevention than on long term health risks particularly those are of 51 years or older [29].

So we need a thoughtful scrutiny of the practice of prophylactic oophorectomy at all ages, for women who are not known to be at increased risk for ovarian cancer. For all women who decide to keep their ovaries, a very strong argument should at least be made for bilateral salpingectomy as this may reduce the chance of subsequent ovarian cancer development.

\section{Conclusion}

The decision regarding elective oophorectomy at time of hysterectomy for benign indications is complex. The approach to such decisions must include comprehensive counseling regarding risks and benefits and shared decision making between the clinician and patient. In women not at high risk for development of ovarian or breast cancer, removing the ovaries at the time of hysterectomy should be approached with caution. Additionally, more research is needed to determine the relative benefits and risks of hormone therapy for this group of women.

\section{References}

[1] Hickey M, Ambekar M, Hammond I. Should the ovaries be removed or retained at the time of hysterectomy for benign disease. Human Reproduction Update 2010;16(2):131-141.

[2] Wu JM, Wechter ME, Geller EJ, Nguyen TV, Visco AG. Hysterectomy rates in the United States 2003. Obstet Gynecol 2007;110:1091-1095.

[3] Bourdrez P, Bongers MY, Mol BW. Treatment of dysfunctional uterine bleeding: patient preferences for endometrial ablation, a levonorgestrel releasing intrauterine device or hysterectomy. Fertil Steril 2004;82: 160-166.

[4] American Cancer Society, Cancer reference information 2009; Available at http://www.cancer.org (Accessed October 20, 2013). 
[5] Whiteman MK, Hillis SD, Jamieson DJ, Morrow B, Podgornik MN, Brett KM et al. Inpatient hysterectomy surveillance in the United States, 2000-2004. Am J Obstet Gynecol 2008; 198(1) 34.e1-34.e7.

[6] Jacoby VL, Vittinghoff E, Nakagawa S, Jackson R, Richter HE, Chan J, et al. Factors associated with undergoing bilateral salpingo-oophorectomy at the time of hysterectomy for benign conditions. Obstet Gynecol. Jun 2009;113(6):1259-67.

[7] Anderson GL, Limacher M, Assaf AR, Bassford T, Beresford SA, Black $\mathrm{H}$, et al. Women's Health Initiative Steering Committee. Effects of conjugated equine estrogen in postmenopausal women with hysterectomy: the Women's Health Initiative randomized controlled trial. JAMA 2004;291 (14) 1701- 1712.

[8] Labrie F, Martel C, Balser J. Wide distribution of the serum dehydroepiandrosteron and sex steroid levels in postmenopausal women: role of the ovary? Menopause 2011; $18: 30-43$

[9] Lasley BL, Crawford SL, Laughlin GA, Santoro N, McConnell DS, Crandall C, et al. Circulating dehydroepiandrosterone sulfate levels in women who underwent bilateral salpingo-oophorectomy during the menopausal transition. Menopause 2011;18:494-8.

[10] Fogle RH, Stanczyk FZ, Zhang X, Paulson RJ. Ovarian androgen production in postmenopausal women. J Clin Endocrinol Metab 2007; 92:3040-43.

[11] Bukovsky I, Halperin R, Schneider D. Ovarian function following abdominal hysterectomy with and without unilateral oophorectomy. Eur J Obstet Gynecol Reprod Biol, 1995; 58(1):29-32.

[12] Jacoby VL, Grady D, Wactawski-Wende J, Manson JE, Allison MA, Kuppermann M, et al. Oophorectomy vs ovarian conservation with hysterectomy: cardiovascular disease, hip fracture and cancer in the Women' $\mathrm{s}$ Health Initiative observational study. Arch Intern Med. 2011; 171(8):760-768.

[13] National cancer Institute. SEER Stat Fact Sheets: Ovary Cancer. Available at http://seer.cancer.gov/statfacts/html/ovary.html (Accessed on September 24, 2013)

[14] 14. Whittemore AS, Harris R, Itnyre J. Characteristics relating to ovarian cancer risk: collaborative analysis of 12 US case -control studies.IV. The pathogenesis of epithelial ovarian cancer. Collaborative Ovarian Cancer Group. Am J Epidemiol 1992; 136:1212.

[15] Parkar WH, Broder MS, Chang E, Feskanich D, Farquhar C, Liu Z, et al. Ovarian conservation at the time of hysterectomy and long term health outcomes in the nurses' health study. Obstet Gynecol 2009; 113:1027-37.

[16] 16. Dekel A, Efrat Z, Orvieto R , Levy T, Dicker D, Gal R et al. The residual ovary syndrome: a 20 -year experience. Eur J Obstet Gynecol Reprod Biol 1996; 68:159-164.

[17] Plockinger B, Kolbl H. Development of ovarian pathology after hysterectomy without oophorectomy. J Am Coll Surg 1994;178:581-5.

[18] Salim R, Gray G, Chappatte OA. The feasibility and efficacy of laparoscopic oophorectomy in the management of pelvic pain after hysterectomy. J Obstet Gynecol 2007; 27 :718-20.

[19] Lobo RA. Surgical menopause and cardiovascular risks. Menopause 2007; 14:562-566.

[20] Kannel WB, Hjortland MC, McNamara PM, Gordon T. Menopause and risk of cardiovascular disease: the Framingham study. Ann Intem Med 1976; 85: 447-452.

[21] Allison MA, Manson JE, Langer RD, Carr JJ, Rossouw JE, Pettinger MB, et al. Oophorectomy, hormone therapy, and subclinical coronary artery disease in women with hysterectomy: the Women's Health Initiative coronary artery calcium study. Women's Health Initiative and Women's Health Initiative Coronary Artery Calcium Study Investigators. Menopause 2008;15:639-47.

[22] Atsma F, Bartelink ML, Grobbee DE, van der Schouw YT. Postmenopausal status and early menopause as independent risk factors for cardiovascular disease: a meta-analysis. Menopause 2006; $13: 1021-1028$.

[23] Lokkegaard E, Jovanovic Z, Heitmann BL, Keiding N, Ottesen B, Pedersen AT. The association between early menopause and risk of ischaemic heart disease: influence of hormone therapy. Maturitas 2006;53:226-233.

[24] Rocca WA, Grossardt BR, Maraganore DM. The long term effects of oophorectomy on cognitive and motor aging are age dependent. Neurodegener Dis 2008; 5:257-260.

[25] Rocca WA, Bower JH, Maraganore DM, Ahlskog JE, Grossardt BR, de Andrade $\mathrm{M}$ et al. Increased risk of parkinsonism in women who underwent oophorectomy before menopause. Neurology 2008; 70(3):200-9.

[26] Hicky M, Davis SR, Sturdee DW. Treatment of menopausal symptoms: what shall we do now? Lancet 2005;366:409-421.

[27] Shuster LT, Gostout BS, Grossardt BR, Rocca WA. Prophylactic oophorectomy in premenopausal women and long term health. Menopause Int 2008; 14:111-116.

[28] Shoupe D, Parker WH, Broder MS, Liu Z, Farquhar C, Berek JS. Elective oophorectomy for benign gynaecological disorders. Menopause 2007; 14:580-5.

[29] Reed SD, Goff B. elective oophorectomy or ovarian conservation at the time of hysterectomy-updated on Oct 19 2012. Available from: http://www.uptodate.com/contents/elective-oophorectomy-o r-ovarian-conservation-at-the-time-of-hysterectomy. (Accessed on September 04, 2013)

[30] Davis SR, Davison SL, Donath S, Bell RJ. Circulating androgen levels and self- reported sexual function in women. JAMA 2005;294:91-96.

[31] Shifren JL, Avis NE. Surgical menopause: effects on psychological well-being and sexuality. Menopause2007;14:586-591.

[32] Nathorst-Boos J, Von-Schoultz B,Carlstrom K. Elective ovarian removal and estrogen replacement therapy-effects on sexual life, psychological well-being and androgen status. J Psychosom Obstet Gynaecol 1993; 14:283-293.

[33] Kotz K, Alexander JL, Dennerstein L. Estrogen and androgen hormone therapy and well-being in surgically post menopausal women. J Womens Health(Larchmt)2006;15:898-908. 
[34] Prospective Studies Collaboration. Age-specific relevance of usual blood pressure to vascular mortality: a meta-analysis of individual data for one million adults in 61 prospective studies. Lancet.2002;360:1903-1913.

[35] Vasan RS, Larson MG, Leip EP, Evans JC, O'Donnell CJ, Kannel WB, et al. Impact of high-normal blood pressure on the risk of cardiovascular disease. $\mathrm{N}$ Engl $\mathrm{J}$ Med. 2001;345:1291-1297.

[36] Li CI, Malone KE, Porter PL, Weiss NS, Tang MT, Cushing-Haugen KL, et al. Relationship between long durations and different regimens of hormone therapy and risk of breast cancer. JAMA.2003;289:3254-3263.

[37] Greendale GA, Reboussin BA, Sie A. Effects of estrogen and estrogen-progestin on mammographic parenchymal density. Ann Intern Med.1999;130:262-269.

[38] FDA News Release. FDA updates hormone therapy information for postmenopausal women. February 10, 2004. Available http://www.fda.gov/bbs/topics/NEWS/2004/NEW01022.ht $\mathrm{ml}$ (Accessed November 5, 2013).

[39] Parker WH, Broder MS, Liu Z, Shoupe D, Farquhar C, Berek JS. Ovarian conservation at the time of hysterectomy for benign disease. Obstet Gynecol 2005; 106:219-26.

[40] ACOG. ACOG Practice Bulletin No. 89. Elective and risk-reducing salpingo-oophorectomy. Obstet Gynecol 2008; 111:231-41. 